\title{
Identification of Knee Osteoarthritis Based on Bayesian Network: Pilot Study
}

Bo Sheng ${ }^{1,2}$, PhD; Liang Huang ${ }^{3}$, PhD; Xiangbin Wang ${ }^{1}, \mathrm{PhD}$; Jie Zhuang ${ }^{4}$, PhD; Lihua Tang ${ }^{2}$, PhD; Chao Deng ${ }^{5}$, $\mathrm{PhD}$; Yanxin Zhang ${ }^{1,3,4}$, PhD

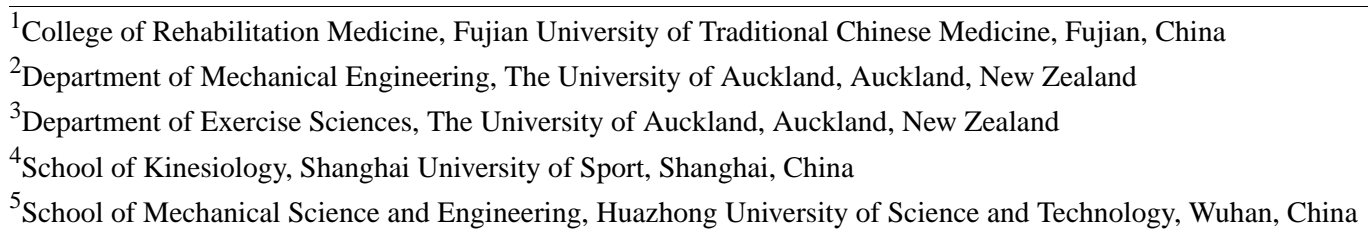

Corresponding Author:

Yanxin Zhang, PhD

Department of Exercise Sciences

The University of Auckland

4703906, Newmarket

Auckland,

New Zealand

Phone: 6499236859

Email: yanxin.zhang@auckland.ac.nz

\section{Abstract}

Background: Early identification of knee osteoarthritis (OA) can improve treatment outcomes and reduce medical costs. However, there are major limitations among existing classification or prediction models, including abstract data processing and complicated dataset attributes, which hinder their applications in clinical practice.

Objective: The aim of this study was to propose a Bayesian network (BN)-based classification model to classify people with knee OA. The proposed model can be treated as a prescreening tool, which can provide decision support for health professionals.

Methods: The proposed model's structure was based on a 3-level BN structure and then retrained by the Bayesian Search (BS) learning algorithm. The model's parameters were determined by the expectation-maximization algorithm. The used dataset included backgrounds, the target disease, and predictors. The performance of the model was evaluated based on classification accuracy, area under the curve (AUC), specificity, sensitivity, positive predictive value (PPV), and negative predictive value (NPV); it was also compared with other well-known classification models. A test was also performed to explore whether physical fitness tests could improve the performance of the proposed model.

Results: A total of 249 elderly people between the ages of 60 and 80 years, living in the Kongjiang community (Shanghai), were recruited from April to September 2007. A total of 157 instances were adopted as the dataset after data preprocessing. The experimental results showed that the results of the proposed model were higher than, or equal to, the mean scores of other classification models: .754 for accuracy, .78 for AUC, .78 for specificity, and .73 for sensitivity. The proposed model provided .45 for PPV and .92 for NPV at the prevalence of $20 \%$. The proposed model also showed a significant improvement when compared with the traditional BN model: $6.3 \%$ increase in accuracy (from .709 to .754), $4.0 \%$ increase in AUC (from .75 to .78), $6.8 \%$ increase in specificity (from .73 to .78), 5.8\% increase in sensitivity (from .69 to .73), 15.4\% increase in PPV (from .39 to .45 ), and $2.2 \%$ increase in NPV (from .90 to .92). Furthermore, the test results showed that the performance of the proposed model could be largely enhanced through physical fitness tests in 3 evaluation indices: $10.6 \%$ increase in accuracy (from .682 to .754 ), $16.4 \%$ increase in AUC (from .67 to .78), and 30.0\% increase in specificity (from .60 to .78).

Conclusions: The proposed model presents a promising method to classify people with knee OA when compared with other classification models and the traditional BN model. It could be implemented in clinical practice as a prescreening tool for knee OA, which would not only improve the quality of health care for elderly people but also reduce overall medical costs.

(JMIR Med Inform 2019;7(3):e13562) doi: 10.2196/13562 


\section{KEYWORDS}

osteoarthritis; knee; classification; health services for the aged; physical fitness; Bayesian network

\section{Introduction}

\section{Background}

Knee osteoarthritis (OA) is a progressive and irreversible condition affecting more than 250 million people around the world $[1,2]$. Early identification of knee OA is important, as it can improve treatment outcomes and reduce medical costs [3]. There are 2 traditional identification methods: imaging-based metrics (eg, x-rays and magnetic resonance imaging [MRI]) and patient-reported metrics (eg, pain). However, imaging-based metrics have some limitations: x-rays are not suitable for pregnant women, MRI is expensive, and both of them lack portability [4]. Meanwhile, patient-reported metrics are subjective and inconsistent [5]. To overcome these limitations, several studies have attempted to develop classification or prediction models to identify knee OA. The key elements of these models are algorithms and dataset attributes. Commonly used algorithms include logistic regression (LR) [2,6,7] and artificial neural network [8]. Commonly used dataset attributes include biometric characteristics $[2,6,7,9,10]$ (eg, age, gender, and body mass index [BMI]) and other medical information $[2,6,7]$ (eg, knee pain, occupational risks, and medical tests scores). The identification accuracy of these models is around $70 \%$. However, there are 2 main issues surrounding these models [11]. First, data processing (reasoning and expression) is hard for both therapists and patients to understand; for example, as data processing within artificial neural networks is encapsulated and abstract, the study of their structures contributes little to their results (eg, there is no simple link between the network topology and the results). Second, the dataset attributes in some studies are too complicated; for example, 1 dataset [10] of 186 attributes contained variables from radiographs (eg, medial alignment angle), as well as biochemical markers from serum and urine (eg, fibulin 3-1), making them difficult and costly to collect.

\section{Research Motivations}

Bayesian network (BN), in contrast, has the advantage of being applicable in classification or prediction models. Because its procedures of reasoning and expression can be easily understood and accepted by both therapists and patients, unlike the black box of other traditional algorithms, it is also able to present uncertainties and causalities, which are both important in the medical domain [12]. Several studies have examined the performance of $\mathrm{BN}$ by developing mathematical models for diagnosing different diseases, including breast cancer [13], lung cancer [14], and Alzheimer disease [12,15]. These experimental results showed that all models of these disease diagnoses provided accuracy of at least $80 \%$, and their network structures could be easily understood. To date, only 1 study has been conducted using the $\mathrm{BN}$ model for the identification of knee OA [5]. Although the model is helpful in identifying the relationship between different risk factors, the practical clinical implications are minimal because the used radiographic data (eg, joint space narrowing) can be directly used to diagnose knee OA even without the model.

On the other hand, researchers reported that the results of simple physical fitness tests could provide useful information to help assess bodily functions or diseases [16-18]. On the basis of the report by Dobson [19], several physical fitness indices have been used to identify knee OA, such as the Timed Up and Go (TUG) test and the 6-min walk test (6MWT). These physical fitness tests have been applied in clinical practice [20,21]. Compared with other biomarkers, physical fitness scores are easily measured using low-cost equipment, making them suitable for community health centers.

\section{Research Purpose}

The main purpose of this research was to propose a BN-based classification model for classifying people with knee OA. Specifically, the proposed BN will be modeled via a combination of expert knowledge and data-oriented modeling. Its network structure will be manually constructed based on a systematic review of literature and experts' opinions, and automatically retrained by the BS learning algorithm [22]. Its network parameters will be learned by the expectation-maximization (EM) algorithm [23], and its dataset attributes will include backgrounds (5 attributes, subjects' basic characteristics), the target disease, and predictors (13 attributes, physical fitness tests scores). The proposed model from this research could be implemented in clinical practice as a prescreening tool for knee OA, which could promote proactive knee OA prevention. The rest of the paper is organized as follows: Methods section details the dataset attributes used for training and validation, and the procedures for building the BN model; Results section presents the experimental result, which is discussed in the Discussion section, followed by the conclusive remarks in Conclusions.

\section{Methods}

\section{Subjects and Data Measurement}

This research used a dataset from a previous study (titled The effectiveness of a combined exercise intervention on physical fitness factors related to falls in community-dwelling older adults [24]), which was approved by Ethics Advisory Committee of Shanghai University of Sport. All participants gave their written informed consent before study. Subjects (aged between 60 and 80 years) were given an orientation (eg, study objectives, risks and benefits, and data collection procedures) and were asked to sign a consent form. The following basic characteristics were then collected from each subject through a questionnaire and a basic measurement: disease condition, gender, age, level of education, height, weight, waist girth, and hip girth. A total of 6 physical fitness tests were conducted after the basic characteristics collection: the single-leg stance balance (SLSB) test, body reaction time (BRT) test, modified sit and reach (MSR) test, leg extension power (LEP) test, TUG test, and Star Excursion Balance Test (SEBT). These tests provide different 
indices of physical fitness and/or activities of daily living for participants (Table 1). Their reliability [25-30] and predictive validity for knee OA have been verified [24,31-34]. The duration of the whole experiment for each subject was approximately 1 hour, and the detailed measurement of these 6 physical fitness tests has been presented in Multimedia Appendix 1.

Table 1. The measurements of 6 physical fitness tests.

\begin{tabular}{llll}
\hline Test & Measurement & Unit & ICC \\
\hline Single-leg stance balance test & Duration of body balance & Seconds & Seconds \\
Body reaction time test & Time of body reaction & Centimeters & .994 [25] \\
Modified sit and reach test & Distance reached by the tip of the fingers & Watts & $.980[27]$ \\
Leg extension power test & Extension power of the leg muscles & Seconds & $.900[28]$ \\
Timed Up and Go test & Time taken to finish the test (go and come back) & Centimeters & $.990[29]$ \\
Star Excursion Balance Test & Distance between both feet (8 directions) & $.990[30]$ \\
\hline
\end{tabular}

${ }^{\mathrm{a}} \mathrm{ICC}$ : intraclass correlation coefficient.

\section{Data Analysis and Preprocessing}

Before constructing the $\mathrm{BN}$ model, the collected data are preprocessed: some original attributes of background information are merged with new attributes, which are more sensitive to knee OA. According to the studies by Zhang [2] and Gandhi [35], BMI and waist-to-hip ratio (WHR) are common risk factors for knee OA, with their predictive validity being well verified. Therefore, in this research, BMI is used instead of height and weight, and WHR is used instead of waist girth and hip girth. Furthermore, Creamer [36] reported that education level is related to knee OA, as it influences the self-reported pain severity of knee OA. Thus, 5 basic characteristics (gender, age, BMI, WHR, and education level) of participants are determined.

According to biostatistics literature [37], data will lose its measure of confidence if its missing value ratio is greater than $30 \%$. Therefore, for our research, some instances were removed from the dataset if they had more than 6 missing attributes ( 6 of 18). These missing attributes are normally caused by time conflicts and failures in the tests. As a result, a total of 131 instances were used as the primary dataset. The missing values of the primary dataset (11 of 2489) were then imputed using a filter commonly used in data mining classification techniques. The filter named ReplaceMissingValues then scanned all the values and replaced the missing values with mean values $[38,39]$. The demographic characteristics of the primary dataset have been presented in Table 2. Furthermore, according to recent literature [12,14], an imbalanced dataset will cause a skewed classification of the predicting target. In other words, the classification model will have high accuracy for the majority class but low accuracy for the minority class. As for our research, the states in the targeted disease are imbalanced: $40.5 \%$ positive cases and $59.5 \%$ negative cases (Table 2). To balance the dataset, the synthetic minority oversampling technique method was used. This method allows oversampling of the positive cases with little change in the characteristic of the primary dataset [40], and it has been used by many researchers to process imbalanced datasets [12,41]. Finally, a total of 157 instances were adopted in the final dataset, which contained $50.3 \%$ positive cases and $49.7 \%$ negative cases. The demographic characteristics of the final dataset are presented in Table 2.

There are 2 types of variables, which can be handled by the BN model: continuous variables and discrete variables. Normally, most BN models will handle discrete variables [12,14,42]. In this research, we also focused on discrete variables for 3 reasons: (1) the results of our model are discrete; (2) the influence of abnormal values could be avoided, thus making the model more robust; and (3) discrete variables provide better interactions with users, as evidence could be easily selected from a set (eg, the user could select good, moderate, or bad from the test results). A simple k-means algorithm was used to cluster and estimate the cutting point of each continuous attribute. All the filters and algorithms are available in WEKA 3.6 (The University of Waikato, Hamilton, Waikato, New Zealand), a popular machine learning software [43]. The discretization results are presented in Table 3, and the procedure for data collection and preprocessing is presented in Figure 1. 
Table 2. The demographic characteristics of the subjects.

\begin{tabular}{|c|c|c|}
\hline Attribute & Primary $(\mathrm{N}=131)$ & Final $(N=157)$ \\
\hline \multicolumn{3}{|l|}{ Gender, n (\%) } \\
\hline Male & $45(34.4)$ & $54(34.4)$ \\
\hline Female & $86(65.6)$ & $103(65.6)$ \\
\hline Age (years), mean (SD) & $70.37(5.70)$ & $70.31(5.56)$ \\
\hline Body mass index $\left(\mathrm{kg} / \mathrm{m}^{2}\right)$, mean (SD) & $25.25(3.89)$ & $25.31(3.74)$ \\
\hline Waist-to-hip ratio, mean (SD) & $0.91(0.08)$ & $0.92(0.08)$ \\
\hline \multicolumn{3}{|l|}{ Education, $\mathrm{n}(\%)$} \\
\hline Junior and below & $38(29.0)$ & $47(29.9)$ \\
\hline Junior high & $41(31.3)$ & $61(38.9)$ \\
\hline Senior high and above & $44(33.6)$ & $49(31.2)$ \\
\hline Missing & $8(6.1)$ & $-^{\mathrm{a}}$ \\
\hline \multicolumn{3}{|l|}{ Osteoarthritis, n (\%) } \\
\hline Negative & $78(59.5)$ & $78(49.7)$ \\
\hline Positive & $53(40.5)$ & $79(50.3)$ \\
\hline \multicolumn{3}{|l|}{ Physical fitness test and unit, mean (SD) } \\
\hline Single-leg stance balance test (eyes open, s) & $72.77(81.84)$ & $70.30(79.93)$ \\
\hline Body reaction time test (s) & $0.63(0.17)$ & $0.64(0.17)$ \\
\hline Modified sit and reach test ( 3 missing, $\mathrm{cm}$ ) & $24.47(9.47)$ & $24.55(9.07)$ \\
\hline Leg extension power test $(\mathrm{w})$ & $287.41(258.30)$ & $289.62(278.33)$ \\
\hline Timed Up and Go test (s) & $8.85(2.02)$ & $8.91(2.02)$ \\
\hline Anterior Star Excursion Balance Test ${ }^{\mathrm{b}}$ & $0.77(0.11)$ & $0.76(0.11)$ \\
\hline Anterolateral Star Excursion Balance Test ${ }^{\mathrm{b}}$ & $0.83(0.10)$ & $0.83(0.10)$ \\
\hline Lateral Star Excursion Balance Test ${ }^{b}$ & $0.81(0.13)$ & $0.81(0.13)$ \\
\hline Posterolateral Star Excursion Balance Test ${ }^{\mathrm{b}}$ & $0.77(0.15)$ & $0.77(0.15)$ \\
\hline Posterior Star Excursion Balance Test ${ }^{\mathrm{b}}$ & $0.67(0.18)$ & $0.66(0.17)$ \\
\hline Posteromedial Star Excursion Balance Test ${ }^{\mathrm{b}}$ & $0.61(0.17)$ & $0.61(0.17)$ \\
\hline Medial Star Excursion Balance Test ${ }^{b}$ & $0.50(0.16)$ & $0.50(0.15)$ \\
\hline Anteromedial Star Excursion Balance Test ${ }^{\mathrm{b}}$ & $0.68(0.11)$ & $0.69(0.11)$ \\
\hline
\end{tabular}

${ }^{\mathrm{a}}$ Data not available.

${ }^{b}$ The measured value for the Star Excursion Balance Test has been normalized (without unit). 
Table 3. The discretization results of the final dataset.

\begin{tabular}{|c|c|}
\hline Level and attribute & States \\
\hline \multicolumn{2}{|l|}{ Background } \\
\hline Gender & 1: male; 2 : female \\
\hline Age (years) & 1: [0 to 70$] ; 2:$ (70 to infinity) \\
\hline Body mass index $\left(\mathrm{kg} / \mathrm{m}^{2}\right)$ & 1: [0 to 25$) ; 2:$ (25 to infinity) \\
\hline Waist-to-hip ratio & 1: [0 to 0.91$] ; 2:$ (0.91 to infinity) \\
\hline Education & 1: junior and below; 2 : junior high; 3 : senior high and above \\
\hline \multicolumn{2}{|l|}{ Disease } \\
\hline Osteoarthritis & 1: negative; 2 : positive \\
\hline \multicolumn{2}{|l|}{ Predictor } \\
\hline Single-leg stance balance test (s) & 1: [0 to 73.6$] ; 2:$ (73.6 to infinity) \\
\hline Body reaction time test (s) & 1: [0 to 0.63$] ; 2:$ (0.63 to infinity) \\
\hline Modified sit and reach test $(\mathrm{cm})$ & 1: [0 to 24.3$] ; 2:$ (24.3 to infinity) \\
\hline Leg extension power test $(\mathrm{w})$ & 1: [0 to 281]; 2: (281 to infinity) \\
\hline Timed Up and Go test (s) & 1: [0 to 8.9$] ; 2:$ ( 8.9 to infinity) \\
\hline Anterior Star Excursion Balance Test & $1:[0$ to 0.763$] ; 2:(0.763 \text { to } 2.00)^{\mathrm{a}}$ \\
\hline Anterolateral Star Excursion Balance Test & 1: [0 to 0.833$] ; 2:(0.833 \text { to } 2.00)^{\mathrm{a}}$ \\
\hline Lateral Star Excursion Balance Test & 1: [0 to 0.812$] ; 2:(0.812 \text { to } 2.00)^{\mathrm{a}}$ \\
\hline Posterolateral Star Excursion Balance Test & 1: [0 to 0.749$] ; 2:(0.749 \text { to } 2.00)^{\mathrm{a}}$ \\
\hline Posterior Star Excursion Balance Test & 1: $[0$ to 0.658$] ; 2:(0.658 \text { to } 2.00)^{\mathrm{a}}$ \\
\hline Posteromedial Star Excursion Balance Test & $1:[0$ to 0.607$] ; 2:(0.607 \text { to } 2.00)^{\mathrm{a}}$ \\
\hline Medial Star Excursion Balance Test & 1: [0 to 0.490$] ; 2:(0.490 \text { to } 2.00)^{\mathrm{a}}$ \\
\hline Anteromedial Star Excursion Balance Test & $1:[0$ to 0.682$] ; 2:(0.682 \text { to } 2.00)^{\mathrm{a}}$ \\
\hline
\end{tabular}

${ }^{\mathrm{a}}$ The measured value for the Star Excursion Balance Test has been normalized. 
Figure 1. Flowchart of the data collection and preprocessing steps.

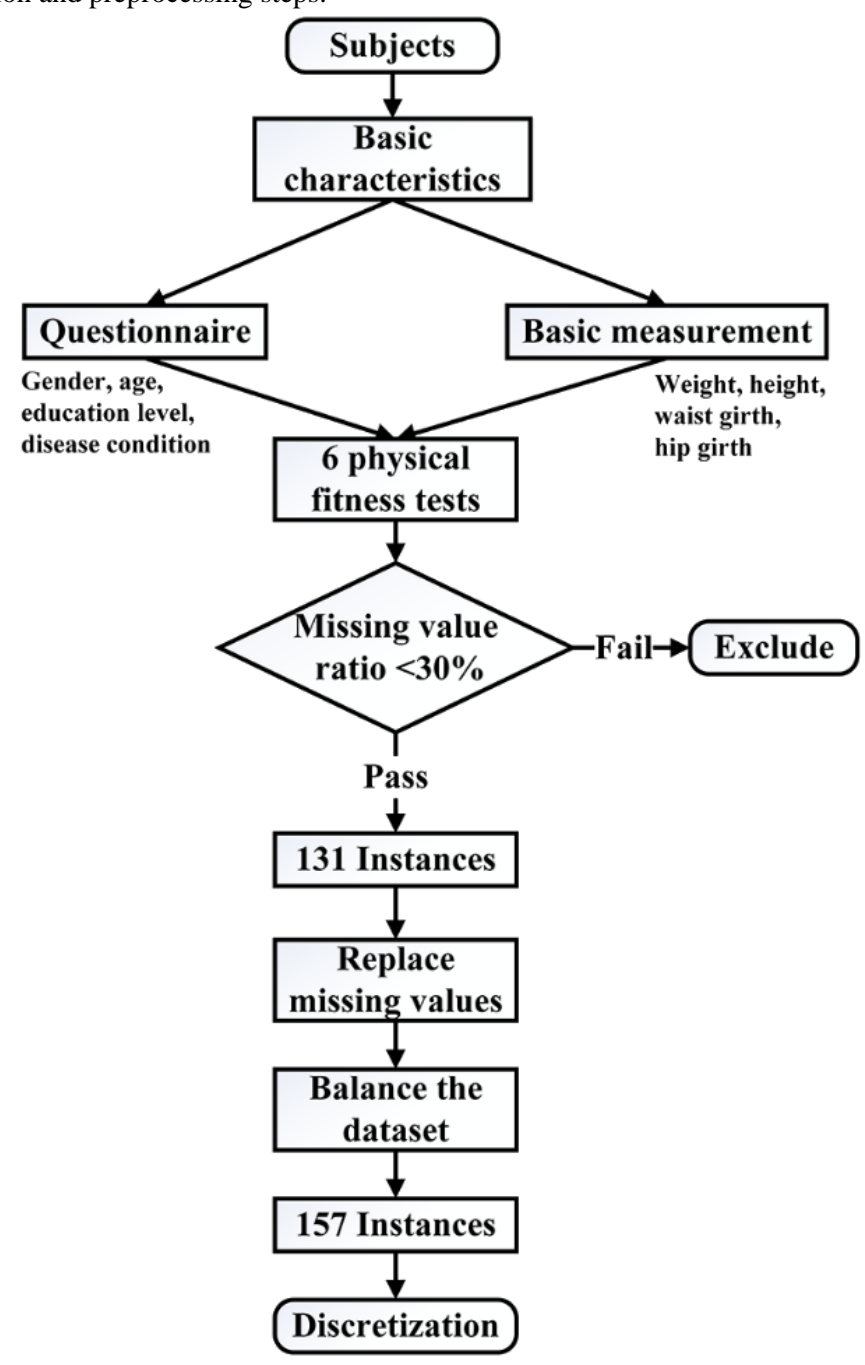

\section{Bayesian Network Concept and Modeling}

The $\mathrm{BN}$ is a probability graphical model, which describes a set of random variables and their conditional dependencies through a directed acyclic graph [44]. The key elements in building a $\mathrm{BN}$ are its structure and parameters. The structure contains nodes and their directed edges: each node expresses a variable of the $\mathrm{BN}$, and each directed edge represents a direct dependency between each pair of nodes. The parameters (conditional probability tables) represent prior knowledge of each node, which can be obtained from experts or specialized learning algorithms. Once the structure and parameters are determined, the results (posterior probability distribution, eg, the percentages of knee OA and not knee OA) of query variables will be calculated by the inference engine each time a user inputs evidence. A simple example of a 3-level BN model, including the background level, target disease level, and predictor level, in the medical domain is shown in Figure 2. The background level contains subjects' basic information such as gender, age, and education; the target disease level shows the predicted disease; and the predictor level presents the predictors, which include signs, symptoms, and the test results. The basic principle of conditional probability is based on Bayes' theorem:

$$
\boldsymbol{P}(\mathrm{A} \mid \mathrm{B})=\frac{\boldsymbol{P}(\mathrm{B} \mid \mathrm{A}) \mathrm{P}(\mathrm{A})}{\mathrm{P}(\mathrm{B})}
$$

where $\mathrm{A}$ and $\mathrm{B}$ are events, and $\mathrm{P}(\mathrm{B}) \neq 0$ [42]. A basic 3-level BN model in the medical domain for the diagnosis of tuberculosis has been attached as Multimedia Appendix 2.

As discussed in the section previously, BN modeling mainly contains 2 tasks: structure learning and parameter learning. During structure learning, we develop a semihandcrafted network structure. The basic structure (Figure 3, the black lines) is constructed according to related knee OA literature [2,3,5,45] and is examined by domain experts. Specifically, 5 basic characteristics (gender, age, BMI, WHR, and education) of participants are set as the background level, knee OA is set as the target disease level, and 6 physical fitness tests (SLSB, BRT, MSR, LEP, TUG tests, and SEBT [it has 8 directions]) are set as the predictor level. As mentioned previously, the selected basic characteristics are commonly used risk factors for knee OA $[2,35,36]$, and the selected physical fitness tests have been verified to be effective in predicting knee OA as well [24,31-34]. Moreover, the basic structure is retrained by the BS learning algorithm based on $30 \%$ of the final dataset to get the improved structure, and some hidden relationships between attributes are found as well (Figure 3, the red lines, will be discussed later). The used BS learning algorithm adopts the classification 
accuracy (k-fold cross-validation method, $k=5$ ) as the scoring function in search for the optimal structure [46]. Meanwhile, the EM algorithm is used for parameter learning based on the rest of the final dataset during validation. This algorithm has the ability to learn parameters of a given $\mathrm{BN}$ structure from the dataset that contains missing values [23]. Furthermore, the clustering algorithm is used as the inference engine because our BN model is simple (a total of 18 attributes). The whole procedure for building the proposed semihandcrafted $\mathrm{BN}$ (SHBN) model has been shown in Figure 4. In this research, the BN toolbox in Matlab 2016b (MathWorks Inc., Natick, MA, USA) was used to determine the structure and parameters, and GeNIe 2.2 (BayesFusion LLC, Pittsburgh, PA, USA) was used as the interface engine to allow users to interact with the BN model and view the results. It should be noted that we kept both the basic handcrafted BN (HBN) and SHBN models to explore whether the performance of the traditional $\mathrm{BN}$ model can be improved by advanced learning algorithms (in the aspect of structure).

Figure 2. Three-level Bayesian network model in the medical domain.

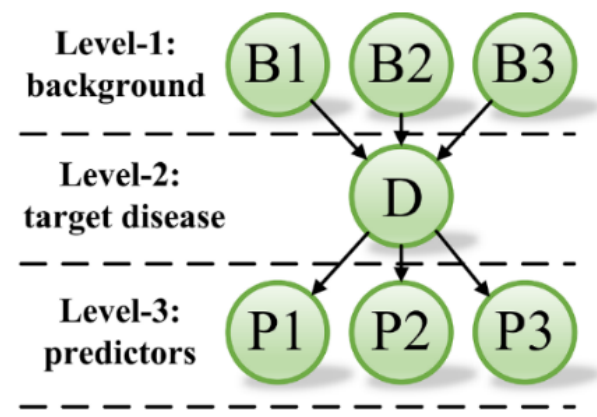

Figure 3. The semihandcrafted Bayesian network model. BMI: body mass index; WHR: waist-to-hip ratio.

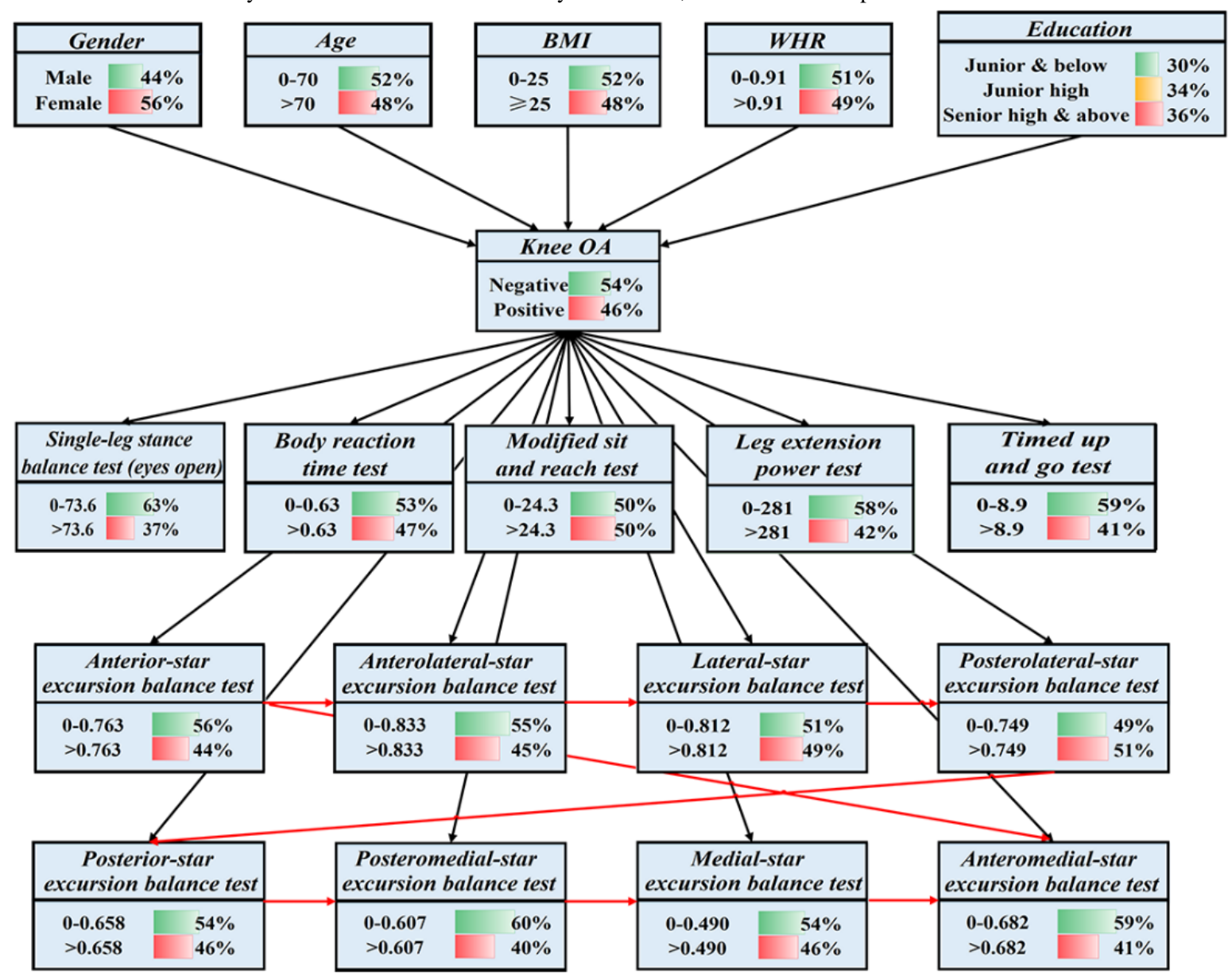


Figure 4. The procedure for building the semihandcrafted Bayesian network model. BS: Bayesian Search; EM: Expectation-Maximization.

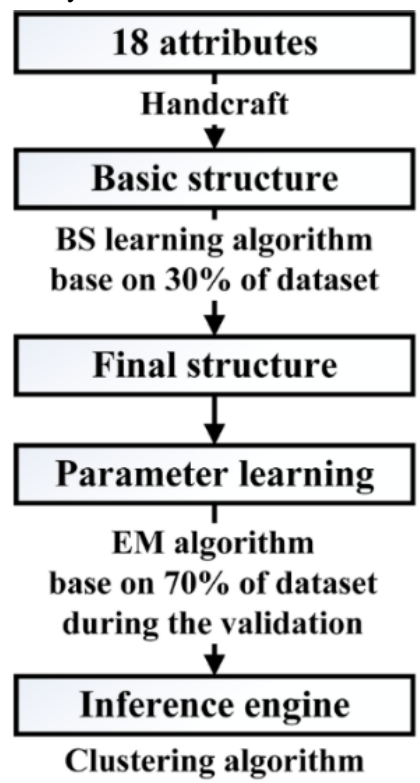

\section{Results}

\section{Model Evaluation Criteria}

The proposed SHBN model is evaluated against 2 criteria: the classification performance and the robustness. The classification performance (eg, classification accuracy and area under the curve [AUC]) evaluates how well the SHBN model differentiates between 2 states: positive or negative of having knee OA. The robustness (eg, specificity and sensitivity) evaluates the SHBN model's ability to handle uncertainty in the output, which could be affected by the evidence from the input. The specificity here can be named the true negative rate. It can reflect the proportion of healthy subjects who are correctly identified as not having the knee OA. The sensitivity here can be named the true positive rate. It can reflect the proportion of sick subjects who are correctly identified as having the knee OA. To verify the classification performance and robustness of the SHBN model, 6 well-known classification models are selected to make comparisons [11]: decision tree (DT), discriminant analysis, LR, support vector machine, k-nearest neighbor (KNN), and ensemble method (discriminant subspaces-based ensemble method). These classification models have been used by many researchers to identify or classify people with knee OA $[2,47,48]$, and the used Ensemble method is known for processing binary classification [49]. The detailed information (kernel and parameter) of these classification models can be seen in Table 4 and is also available in the Classification Learner App, MATLAB. Furthermore, to explore whether the physical fitness tests could improve the performance of the SHBN model, a test was conducted based only on the subjects' basic characteristics, including gender, age, education level, BMI, and WHR. The k-fold cross-validation method was used for all models based on $70 \%$ of the final dataset $(k=5$, and the other $30 \%$ was specifically used to train the BN structure as described above). The experimental results have been shown in Table 5. On the other hand, knee OA is a condition with increased prevalence. It is necessary to compare the positive predictive value (PPV) and negative predictive value (NPV) of each model according to an apriority probability (the prevalence of knee OA) of $1 \%, 10 \%$, and $20 \%$ [50]. The PPV here means the ability of a model to detect the presence of disease. The NPV here means the ability of a model to detect the absence of disease. PPV and NPV are of high interest for clinical applications; the experimental results are presented in Tables 6 and 7.

\section{Experimental Results}

A total of 249 elderly people aged between 60 and 80 years, living in the Kongjiang community (Shanghai), were recruited from April to September 2007. The ethical approval was obtained from the Ethics Advisory Committee of Shanghai University of Sport. After data preprocessing, a total of 157 instances were adopted as the dataset, which included backgrounds (5 attributes, the basic characteristics of subjects), the target disease (namely the knee OA), and predictors (13 attributes, the scores of physical fitness tests). Table 5 showed that the proposed SHBN model presented a promising result when compared with other classification models, and the scores for all evaluation indices were higher (or equal) than the mean scores. Specifically, based on the criteria of classification performance, (1) for classification accuracy, the Ensemble model received the highest score (.773) followed by the SHBN model (.754) and (2) for the AUC, the Ensemble and LR models received the highest score (.81), whereas the SHBN model (.78) ranked third with little difference with the other scores. On the basis of the criteria of robustness, (1) for specificity, the Ensemble, LR, and SHBN models received the highest score (.78) and (2) for sensitivity, the DT and KNN models received the highest score (.78), whereas the SHBN model (.73) ranked fourth. It should be noted that the HBN model showed a moderate result, and no evaluation indices were better than the mean scores. 
Table 4. Detailed information of well-known classification models.

\begin{tabular}{|c|c|}
\hline Model and kernel & Parameter \\
\hline \multicolumn{2}{|l|}{ Decision tree } \\
\hline Medium tree & Maximum number of splits: 20; Split criterion: Gini's diversity index \\
\hline \multicolumn{2}{|l|}{ Discriminant analysis } \\
\hline Quadratic discriminant & Regularization: diagonal covariance \\
\hline \multicolumn{2}{|l|}{ Logistic regression } \\
\hline Fitlm function & Chi-square statistic versus constant model: 65.1 \\
\hline \multicolumn{2}{|l|}{ Support vector machine } \\
\hline Gaussian $\mathrm{SVM}^{\mathrm{a}}$ & Kernel scale: 2.2; Box constraint level: 1 ; Multiclass method: 1 versus 1 \\
\hline \multicolumn{2}{|l|}{ K-nearest neighbor } \\
\hline Medium $\mathrm{KNN}^{\mathrm{b}}$ & Number of neighbors: 10; Distance metric: Euclidean; Distance weight: equal \\
\hline \multicolumn{2}{|l|}{ Ensemble method } \\
\hline Subspace with discriminant learner & Number of learner: 30; Subspace dimension: 3 \\
\hline
\end{tabular}

${ }^{\mathrm{a}} \mathrm{SVM}$ : support vector machine.

${ }^{\mathrm{b}} \mathrm{KNN}$ : k-nearest neighbor.

Table 5. The performance of models with different criteria.

\begin{tabular}{lllll}
\hline Model & Accuracy & Area under the curve & Specificity & Sensitivity \\
\hline Semihandcrafted Bayesian network & .754 & .78 & .78 & .73 \\
Handcrafted Bayesian network & .709 & .75 & .73 & .69 \\
Decision tree & .736 & .77 & .69 & .78 \\
Discriminant analysis & .709 & .75 & .73 & .69 \\
Logistic regression & .736 & .81 & .78 & .69 \\
Support vector machine & .709 & .77 & .73 & .69 \\
K-nearest neighbor & .727 & .78 & .67 & .78 \\
Ensemble method & .773 & .81 & .78 & .76 \\
Mean score & .732 & .78 & .73 & .73 \\
Semihandcrafted Bayesian network & .682 & .67 & .60 & .76 \\
Logistic regression $^{\mathrm{b}}$ & .709 & .74 & .73 & .69 \\
\hline
\end{tabular}

${ }^{\mathrm{a}}$ The mean score includes the results of the DT, DA, LR, SVM, KNN, and Ensemble method models.

${ }^{\mathrm{b}}$ These results are based only on the subjects' basic characteristics, without the scores of physical fitness tests. 
Table 6. The positive predictive values of models in different conditions.

\begin{tabular}{|c|c|c|c|}
\hline \multirow[t]{2}{*}{ Model } & \multicolumn{3}{|c|}{ The apriority probability } \\
\hline & $1 \%$ & $10 \%$ & $20 \%$ \\
\hline Semihandcrafted Bayesian network & .03 & .27 & .45 \\
\hline Handcrafted Bayesian network & .03 & .22 & .39 \\
\hline Decision tree & .02 & .22 & .39 \\
\hline Discriminant analysis & .03 & .22 & .39 \\
\hline Logistic regression & .03 & .26 & .44 \\
\hline Support vector machine & .03 & .22 & .39 \\
\hline K-nearest neighbor & .02 & .21 & .37 \\
\hline Ensemble method & .03 & .28 & .46 \\
\hline
\end{tabular}

Table 7. The negative predictive values of models in different conditions.

\begin{tabular}{|c|c|c|c|}
\hline \multirow[t]{2}{*}{ Model } & \multicolumn{3}{|c|}{ The apriority probability } \\
\hline & $1 \%$ & $10 \%$ & $20 \%$ \\
\hline Semihandcrafted Bayesian network & 1.00 & .96 & .92 \\
\hline Handcrafted Bayesian network & 1.00 & .95 & .90 \\
\hline Decision tree & 1.00 & .97 & .93 \\
\hline Discriminant analysis & 1.00 & .95 & .90 \\
\hline Logistic regression & 1.00 & .96 & .91 \\
\hline Support vector machine & 1.00 & .95 & .90 \\
\hline K-nearest neighbor & 1.00 & .96 & .92 \\
\hline Ensemble method & 1.00 & .97 & .93 \\
\hline
\end{tabular}

Furthermore, the results of the test showed that the physical fitness tests improved the performance of the classification models, especially for our SHBN model. Specifically, without the attributes of physical fitness tests, the identification accuracy of the SHBN model decreased from .754 to .682, the AUC score decreased from .78 to .67 , the specificity score decreased from .78 to .60 , but the sensitivity score increased from .73 to .76 . The result from the LR model followed a similar trend: the identification accuracy decreased from .736 to .709 , the AUC score decreased from .81 to .74 , and the specificity score decreased from .78 to .73 . The sensitivity score stayed the same (.69). In addition, the results of PPV (Table 6) showed that the Ensemble model received the highest scores in all conditions followed by the SHBN model. The results of NPV (Table 7) presented a similar trend that the Ensemble and DT models received the highest scores in all conditions, whereas the SHBN model received moderate scores in all conditions. It is worth noting that the HBN model ranked fourth for PPV, whereas it ranked last for NPV.

\section{Discussion}

\section{Principal Findings}

The main findings of this research are as follows: (1) the proposed SHBN model presents satisfactory performance to classify people with knee OA in all evaluation indices (accuracy, AUC, specificity, sensitivity, PPV, and NPV); and (2) the proposed SHBN model presents a significant improvement in all evaluation indices when compared with the traditional BN model.

The performance of the SHBN model have been discussed: (1) comparisons with other well-known classification models; (2) comparisons with other BN-based models; and (3) comparisons with traditional HBN model.

First, the performance of each model has been shown in Table 5. Specifically, the SHBN model provided the best specificity (.78), which was the same as the LR and Ensemble models, whereas the highest classification accuracy was achieved by the Ensemble model (.773), the highest AUC was achieved by the LR and Ensemble models (.81), and the best sensitivity was achieved by the DT and KNN models (.78). These results are similar to the research of Seixas [12], in which the BN model did not show the best result as well. The possible reason for this could be that the Ensemble model combines multiple models (eg, subspace analysis and discriminant learner), which produces better performance than a single model [51]. Meanwhile, the BN model has its own shortcomings: some complicated scoring functions require reliable prior knowledge to find a structure that is closer to the realistic model [11]. In this research, the final structure was trained based on the $30 \%$ of the dataset, which could not cover all instances. The reason for not using the whole dataset in the learning of structure and parameter is that it might cause overfitting by using the same dataset to do the cross-validation [52]. In fact, during structure learning, the 
k-fold cross-validation method was used as the scoring function in searching for the optimal structure. In other words, all the results were tested by the cross-validation method. In addition, in terms of PPV and NPV, the SHBN model showed a promising result. Specifically, for PPV (Table 6), the SHBN model received $.03, .27$, and .45 with the apriority probability (the prevalence of knee OA) of $1 \%, 10 \%$, and $20 \%$, respectively. For NPV (Table 7), the SHBN model received 1.00, .96, and .92 with the same trend of the apriority probability. These results are slightly better than the results reported by Peat [53]: .44 for PPV and .72 for NPV with the DT method at the prevalence of $30 \%$. In addition, data from Tables 6 and 7 indicated a trend that PPV and NPV vary with increased prevalence for all models. In other words, in a dataset with higher prevalence of knee OA, PPV increased and NPV decreased, which is supported by Peat [53] as well.

Second, as discussed in the Introduction section, the BN can provide above $80 \%$ accuracy for identifying other diseases. Although knee OA is different from these diseases, 3 possible reasons for the imperfect identification accuracy of our SHBN model were hypothesized. (1) The used dataset was not complicated (only contained 18 attributes), and these attributes came from general information including the basic characteristics of subjects and simple physical fitness scores, rather than special radiographic data such as joint space narrowing. The main reason for using such dataset is to achieve one of the purposes of this research, that is, to develop a classification model for knee OA, which could be easily performed by normal operators, and the used dataset attributes could be collected by cheap and portable equipment, no matter in community health centers or rural hospitals. Therefore, special radiographic data could not be included despite being able to largely improve the performance of the proposed model. (2) The used dataset was not large $(\mathrm{N}=157)$, and there is no doubt that the identification accuracy would be enhanced if a larger dataset is used instead, for example, Wang [14] adopted 4555 instances and achieved .82 accuracy. (3) The skewed dataset might have an impact on the performance, which is suggested by Watt [5], for example, the females covered $66 \%$ of total instances (Table 2). However, because gender is an attribute rather than the target node, it should not be balanced.

Third, the performance of the traditional HBN model across the different evaluation indices was lower than the mean score and of other classification models (Table 5). The results of NPV were also worse than those of other classification models (Table 7). Possible reasons could be similar to that of the SHBN model in which the used attributes were not complicated enough and the dataset was not large enough. However, the SHBN model presented a significant improvement in all evaluation indices when compared with the HBN model: the percent gains for the identification accuracy, the AUC score, the specificity score, the sensitivity score, the PPV, and the NPV were $6.3 \%$ (from .709 to .754 ), $4.0 \%$ (from .75 to .78 ), $6.8 \%$ (from .73 to .78 ), $5.8 \%$ (from .69 to .73), $15.4 \%$ (from .39 to .45, at the prevalence of $20 \%$ ), and $2.2 \%$ (from .90 to .92 , at the prevalence of $20 \%$ ), respectively. A possible reason for this has been explained by Watt [5], where the subjectivity of the handcrafted network structure could bring bias into the modeled BN relations. Due to this, alternative method should be used to automatically suggest the network structure from the dataset. Moreover, Seixas [12] reported a similar finding in which the $\mathrm{BN}$ model discovered from a dataset revealed a slight improvement in some evaluation indices. In that research, the structure of the model was automatically built by the learning algorithm but was problematic because it treated the symptoms as risk factors of the disease, which are incorrect for the diagnosis criteria. Therefore, our research combines the traditional handcrafted approach and the learning algorithm to address this problem (which is why the structure is named semihandcrafted). The final structure of the SHBN can be seen in Figure 3, in which several hidden relationships (red lines) between the 8 directions of SEBT are discovered. It is acceptable that there are correlations between these directions because they belong to the same physical fitness test. In other words, if the result of anterior direction is high, there is a great probability of other directions' results to be high. Meanwhile, no correlation has been found among other physical fitness tests because all of them are independent of each other. It should be noted that if the used dataset is to be changed, the discovered structure may be changed as well. However, in this research, we want to show the possibility that the traditional HBN model can be improved, which has been well verified by the experimental results in all evaluation indices. On the other hand, in fact, no structure can be treated as a one-for-all structure; the practical BN model should be adjusted to meet different requirements of users.

Although the performance of the SHBN model is not the best for all evaluation indices, it still has some advantages in the identification of knee OA. (1) The proposed model has the ability to graphically present the procedures of reasoning and expression, which can help therapists and patients to understand the diagnosis criteria. (2) Due to the used 3-level structure, the proposed model can provide a clearer human-oriented diagram than that of traditional BN models [54]. (3) The proposed model is robust when facing missing values and will create the best possible result with whatever evidence is inputted (dataset with missing values, unfortunately, is the typical case in the medical domain). For example, if 1 subject cannot finish the MSR test and TUG test, the therapist can still use the remaining 16 attributes to identify the knee OA (example for predicting knee OA with missing values has been attached as Multimedia Appendix 3). In addition, the effectiveness of the physical fitness tests is confirmed by the results. Table 5 showed that the identification accuracy of the SHBN model increased from .682 to .754 (percent gain: $10.6 \%$ ), which was similar for the AUC score (from .67 to .78 , percent gain: $16.4 \%$ ) and specificity score (from .60 to .78 , percent gain: $30.0 \%$ ). The performance of the LR model was also improved but was not very obvious when compared with the SHBN model: the percent gains for the identification accuracy, the AUC score, and the specificity score were $3.8 \%$ (from .709 to .736), $9.5 \%$ (from .74 to .81), and $6.8 \%$ (from .73 to .78 ), respectively. A similar result was reported by Zhang [2], in which risk prediction models were developed for knee OA based on LR model, and some basic biometric characteristics (age, gender, BMI, and so on) were used as the predictors. Around .75 of the AUC were calculated by these risk prediction models, which is almost the same result 
as the LR model (.74) in our test using the attributes of subjects' basic characteristics.

In general, the performance of the proposed SHBN model is promising and satisfactory when compared with other well-known models and other BN models, which reveals a good identification result. Meanwhile, the SHBN model shows a significant improvement in all evaluation indices when compared with the HBN model, which confirms that the reliability and validity of the traditional HBN model can be improved by advanced learning algorithms.

\section{Potential Clinical Significance and Future Work}

As discussed in the Introduction section, early identification of knee OA is important to support the timely adjustment of appropriate clinical interventions. In this research, several commonly used basic characteristics of subjects were adopted as inputs for our model to overcome issues that hinder the identification of knee OA, for example, the frequent use of expensive diagnosis tools and special equipment. Meanwhile, to improve the performance of our model, the scores of 6 physical fitness tests were used as the inputs as well. These 6 physical fitness tests can be easily performed in community health centers, and the required equipment is cheap and portable. There are also some advantages in using the $\mathrm{BN}$ model in the medical domain [55] such as adaptability and strong robustness against missing values. Regarding adaptability, the BN model can be started with small and limited domain knowledge and then further extended (or simplified) by inputting new knowledge to suit different requirements. In practice, therapists can collect the up-to-date knowledge of each patient, and the probabilities in the $\mathrm{BN}$ model will be adjusted automatically. Regarding strong robustness against missing values, as discussed in the previous section, the $\mathrm{BN}$ model does not require complete knowledge of the instance and can use as much knowledge as available to do the predication.

In addition, 1 important clinical implication is that the proposed SHBN model can potentially be used as a cheap and portable prescreening tool to identify people with a high risk of knee OA. These identified people are then recommended to undergo further examination using traditional diagnosis tools (eg, $\mathrm{x}$-rays and MRI). The successful identification and treatment of people with knee OA are beneficial for them and the government's health care system because it can reduce long-term morbidity and overall medical costs [2]. Furthermore, the proposed SHBN model can also make the identification of knee OA easier, leading to the better quality of health care for elderly people.

\section{Limitations}

This research has 2 limitations. First, the used dataset was not large $(\mathrm{N}=157)$, and it was not a random sample of the general population. Participants were all elderly people (aged between 60 and 80 years), and most of them resided in the Kongjiang community (Shanghai, China); therefore, the generalizability of the proposed model might be limited. Second, the disease condition of knee OA was self-reported, and the proposed SHBN model could only be treated as the classification model because the used dataset was extracted from the existing data. This warrants future work to overcome the limitations and improve the performance of the proposed model for processing new data by (1) recruiting more subjects with different age and locations to improve the generalizability of the proposed model and (2) including other physical fitness tests for other population groups.

\section{Conclusions}

This paper proposes an SHBN model for the identification of knee OA. This model is based on a 3-level BN structure where background information, target disease, and predictors are linked using hierarchically structured random variables. A total of 157 instances with 18 attributes were used to constitute the subjects' dataset, which included the basic characteristics of subjects and the scores of 6 physical fitness tests. The experimental results showed that the proposed SHBN model can provide a promising and satisfactory result in terms of classification performance (classification accuracy=.754 and AUC=.78), model's robustness (specificity $=.78$ and sensitivity=.73), and predictive performance (PPV=.45 and NPV=.92 at the prevalence of 20\%). In addition to this, the proposed SHBN model represents potential clinical significance because of its advantages, which can be used with appropriate prevention methods to reduce the risk of knee OA in elderly people and improve their quality of health care.

\section{Acknowledgments}

The authors would like to thank Yuanbin Wang and Hao Chen for the technical support and Yujiao Qiao, Peter Fermin Dajime, and Sabina Nanna Yang for manuscript proofreading. The research was supported by the International S\&T Cooperation Program of China (ISTCP) under grant no. 2016YFE0121700, the Cooperation Program of Fujian Key Laboratory of Rehabilitation Technology and Fujian Provincial Rehabilitation Industrial Institution under grant no. 2015Y2001-65, and the Science and Technology Development Fund of Macao SAR (FDCT) under MoST-FDCT joint grant no. 015/2015/AMJ.

\section{Conflicts of Interest}

None declared.

\section{Multimedia Appendix 1}

Detailed measurement for 6 physical fitness tests. 


\section{Multimedia Appendix 2}

A basic 3-level Bayesian network model for the diagnosis of tuberculosis.

[PDF File (Adobe PDF File), 289KB-Multimedia Appendix 2]

\section{Multimedia Appendix 3}

The diagnostic procedure for missing values.

[PDF File (Adobe PDF File), 725KB-Multimedia Appendix 3]

\section{References}

1. Vos T, Flaxman AD, Naghavi M, Lozano R, Michaud C, Ezzati M, et al. Years lived with disability (YLDs) for 1160 sequelae of 289 diseases and injuries 1990-2010: a systematic analysis for the global burden of disease study 2010. Lancet 2012 Dec 15;380(9859):2163-2196 [FREE Full text] [doi: 10.1016/S0140-6736(12)61729-2] [Medline: 23245607]

2. Zhang W, McWilliams DF, Ingham SL, Doherty SA, Muthuri S, Muir KR, et al. Nottingham knee osteoarthritis risk prediction models. Ann Rheum Dis 2011 Sep;70(9):1599-1604. [doi: 10.1136/ard.2011.149807] [Medline: 21613308]

3. Plotnikoff R, Karunamuni N, Lytvyak E, Penfold C, Schopflocher D, Imayama I, et al. Osteoarthritis prevalence and modifiable factors: a population study. BMC Public Health 2015 Nov 30;15:1195 [FREE Full text] [doi: 10.1186/s12889-015-2529-0] [Medline: 26619838]

4. LaValley MP, McLaughlin S, Goggins J, Gale D, Nevitt MC, Felson DT. The lateral view radiograph for assessment of the tibiofemoral joint space in knee osteoarthritis: its reliability, sensitivity to change, and longitudinal validity. Arthritis Rheum 2005 Nov;52(11):3542-3547 [FREE Full text] [doi: 10.1002/art.21374] [Medline: 16255043]

5. Watt EW, Bui AA. Evaluation of a dynamic bayesian belief network to predict osteoarthritic knee pain using data from the osteoarthritis initiative. AMIA Annu Symp Proc 2008 Nov 6:788-792 [FREE Full text] [Medline: 18999030]

6. Takahashi H, Nakajima M, Ozaki K, Tanaka T, Kamatani N, Ikegawa S. Prediction model for knee osteoarthritis based on genetic and clinical information. Arthritis Res Ther 2010;12(5):R187 [FREE Full text] [doi: 10.1186/ar3157] [Medline: $\underline{20939878]}$

7. Kerkhof HJ, Bierma-Zeinstra SM, Arden NK, Metrustry S, Castano-Betancourt M, Hart DJ, et al. Prediction model for knee osteoarthritis incidence, including clinical, genetic and biochemical risk factors. Ann Rheum Dis 2014 Dec;73(12):2116-2121. [doi: 10.1136/annrheumdis-2013-203620] [Medline: 23962456]

8. Yoo TK, Kim DW, Choi SB, Oh E, Park JS. Simple scoring system and artificial neural network for knee osteoarthritis risk prediction: a cross-sectional study. PLoS One 2016;11(2):e0148724 [FREE Full text] [doi: 10.1371/journal.pone.0148724] [Medline: 26859664]

9. Kotti M, Duffell LD, Faisal AA, McGregor AH. Detecting knee osteoarthritis and its discriminating parameters using random forests. Med Eng Phys 2017;43:19-29 [FREE Full text] [doi: 10.1016/j.medengphy.2017.02.004] [Medline: 28242181]

10. Lazzarini N, Runhaar J, Bay-Jensen AC, Thudium CS, Bierma-Zeinstra SM, Henrotin Y, et al. A machine learning approach for the identification of new biomarkers for knee osteoarthritis development in overweight and obese women. Osteoarthritis Cartilage 2017 Dec;25(12):2014-2021 [FREE Full text] [doi: 10.1016/j.joca.2017.09.001] [Medline: 28899843]

11. Linoff GS, Berry MJ. Data Mining Techniques: For Marketing, Sales, And Customer Relationship Management. Hoboken, NJ: John Wiley \& Sons; 2011.

12. Seixas FL, Zadrozny B, Laks J, Conci A, Saade MD. A Bayesian network decision model for supporting the diagnosis of dementia, Alzheimer s disease and mild cognitive impairment. Comput Biol Med 2014 Aug;51:140-158 [FREE Full text] [doi: 10.1016/j.compbiomed.2014.04.010] [Medline: 24946259]

13. Kahn Jr CE, Roberts LM, Shaffer KA, Haddawy P. Construction of a Bayesian network for mammographic diagnosis of breast cancer. Comput Biol Med 1997 Jan;27(1):19-29. [doi: 10.1016/S0010-4825(96)00039-X] [Medline: 9055043]

14. Wang KJ, Makond B, Wang KM. Modeling and predicting the occurrence of brain metastasis from lung cancer by Bayesian network: a case study of Taiwan. Comput Biol Med 2014 Apr;47:147-160. [doi: 10.1016/j.compbiomed.2014.02.002] [Medline: 24607682]

15. Guerrero JM, Martínez-Tomás R, Rincón M, Peraita H. Diagnosis of cognitive impairment compatible with early diagnosis of Alzheimer's disease. A Bayesian network model based on the analysis of oral definitions of semantic categories. Methods Inf Med 2016;55(1):42-49. [doi: 10.3414/ME14-01-0071] [Medline: 25925692]

16. Williams PT. Physical fitness and activity as separate heart disease risk factors: a meta-analysis. Med Sci Sports Exerc 2001 May;33(5):754-761 [FREE Full text] [doi: 10.1097/00005768-200105000-00012] [Medline: 11323544]

17. Rikli RE, Jones CJ. Development and validation of a functional fitness test for community-residing older adults. J Aging Phys Act 1999 Apr;7(2):129-161. [doi: 10.1123/japa.7.2.129]

18. Shumway-Cook A, Silver IF, LeMier M, York S, Cummings P, Koepsell TD. Effectiveness of a community-based multifactorial intervention on falls and fall risk factors in community-living older adults: a randomized, controlled trial. $\mathrm{J}$ Gerontol A Biol Sci Med Sci 2007 Dec;62(12):1420-1427. [doi: 10.1093/gerona/62.12.1420] [Medline: 18166695] 
19. Dobson F, Hinman RS, Hall M, Terwee CB, Roos EM, Bennell KL. Measurement properties of performance-based measures to assess physical function in hip and knee osteoarthritis: a systematic review. Osteoarthritis Cartilage 2012 Dec;20(12):1548-1562 [FREE Full text] [doi: 10.1016/j.joca.2012.08.015] [Medline: 22944525]

20. French HP, Fitzpatrick M, FitzGerald O. Responsiveness of physical function outcomes following physiotherapy intervention for osteoarthritis of the knee: an outcome comparison study. Physiotherapy 2011 Dec;97(4):302-308. [doi: 10.1016/j.physio.2010.03.002] [Medline: 22051586]

21. Stratford PW, Kennedy DM, Woodhouse LJ. Performance measures provide assessments of pain and function in people with advanced osteoarthritis of the hip or knee. Phys Ther 2006 Nov;86(11):1489-1496. [doi: 10.2522/ptj.20060002] [Medline: 17079748 ]

22. Cooper GF, Herskovits E. A Bayesian method for the induction of probabilistic networks from data. Mach Learn 1992 Oct;9(4):309-347. [doi: 10.1007/BF00994110]

23. Moon TK. The expectation-maximization algorithm. IEEE Signal Process Mag 1996 Nov;13(6):47-60. [doi: $10.1109 / 79.543975]$

24. Zhuang J, Huang L, Wu Y, Zhang Y. The effectiveness of a combined exercise intervention on physical fitness factors related to falls in community-dwelling older adults. Clin Interv Aging 2014;9:131-140 [FREE Full text] [doi: 10.2147/CIA.S56682] [Medline: 24453483]

25. Springer BA, Marin R, Cyhan T, Roberts H, Gill NW. Normative values for the unipedal stance test with eyes open and closed. J Geriatr Phys Ther 2007;30(1):8-15. [Medline: 19839175]

26. Eckner JT, Whitacre RD, Kirsch NL, Richardson JK. Evaluating a clinical measure of reaction time: an observational study. Percept Mot Skills 2009 Jun;108(3):717-720. [doi: 10.2466/PMS.108.3.717-720] [Medline: 19725308]

27. Lemmink KA, Kemper HC, de Greef MH, Rispens P, Stevens M. The validity of the sit-and-reach test and the modified sit-and-reach test in middle-aged to older men and women. Res Q Exerc Sport 2003 Sep;74(3):331-336. [doi: 10.1080/02701367.2003.10609099] [Medline: 14510299]

28. Behm DG, Bambury A, Cahill F, Power K. Effect of acute static stretching on force, balance, reaction time, and movement time. Med Sci Sports Exerc 2004 Aug;36(8):1397-1402. [doi: 10.1249/01.MSS.0000135788.23012.5F] [Medline: 15292749]

29. Alghadir A, Anwer S, Brismée JM. The reliability and minimal detectable change of timed up and go test in individuals with grade 1-3 knee osteoarthritis. BMC Musculoskelet Disord 2015 Jul 30;16:174 [FREE Full text] [doi: 10.1186/s12891-015-0637-8] [Medline: 26223312]

30. Plisky PJ, Gorman PP, Butler RJ, Kiesel KB, Underwood FB, Elkins B. The reliability of an instrumented device for measuring components of the star excursion balance test. N Am J Sports Phys Ther 2009 May;4(2):92-99 [FREE Full text] [Medline: 21509114]

31. Maly MR, Costigan PA, Olney SJ. Determinants of self-report outcome measures in people with knee osteoarthritis. Arch Phys Med Rehabil 2006 Jan;87(1):96-104. [doi: 10.1016/j.apmr.2005.08.110] [Medline: 16401446]

32. Huang MH, Lin YS, Yang RC, Lee CL. A comparison of various therapeutic exercises on the functional status of patients with knee osteoarthritis. Semin Arthritis Rheum 2003 Jun;32(6):398-406. [doi: 10.1053/sarh.2003.50021] [Medline: 12833248]

33. Hunt MA, McManus FJ, Hinman RS, Bennell KL. Predictors of single-leg standing balance in individuals with medial knee osteoarthritis. Arthritis Care Res (Hoboken) 2010 Apr;62(4):496-500 [FREE Full text] [doi: 10.1002/acr.20046] [Medline: 20391504]

34. Wegener L, Kisner C, Nichols D. Static and dynamic balance responses in persons with bilateral knee osteoarthritis. J Orthop Sports Phys Ther 1997 Jan;25(1):13-18. [doi: 10.2519/jospt.1997.25.1.13] [Medline: $\underline{8979171]}$

35. Gandhi R, Dhotar H, Tsvetkov D, Mahomed NN. The relation between body mass index and waist-hip ratio in knee osteoarthritis. Can J Surg 2010 Jun;53(3):151-154 [FREE Full text] [Medline: 20507785]

36. Creamer P, Lethbridge-Cejku M, Hochberg MC. Determinants of pain severity in knee osteoarthritis: effect of demographic and psychosocial variables using 3 pain measures. J Rheumatol 1999 Aug;26(8):1785-1792. [Medline: 10451078]

37. Rosner B. Fundamentals Of Biostatistics, 8th Edition. Boston, MA: Cengage Learning; 2016.

38. Smith TC, Frank E. Introducing machine learning concepts with WEKA. Methods Mol Biol 2016;1418:353-378. [doi: 10.1007/978-1-4939-3578-9 17] [Medline: 27008023]

39. Flannery M, Budden DM, Mendes A. FlexDM: simple, parallel and fault-tolerant data mining using WEKA. Source Code Biol Med 2015;10:13 [FREE Full text] [doi: 10.1186/s13029-015-0045-3] [Medline: 26579209]

40. Blagus R, Lusa L. SMOTE for high-dimensional class-imbalanced data. BMC Bioinformatics 2013 Mar 22;14:106 [FREE Full text] [doi: 10.1186/1471-2105-14-106] [Medline: 23522326]

41. Fotouhi S, Asadi S, Kattan MW. A comprehensive data level analysis for cancer diagnosis on imbalanced data. J Biomed Inform 2019 Feb;90:103089. [doi: 10.1016/j.jbi.2018.12.003] [Medline: $\underline{30611011}$ ]

42. Wang Y, Blache R, Zheng P, Xu X. A knowledge management system to support design for additive manufacturing using Bayesian networks. J Mech Des 2018 Mar 14;140(5):051701. [doi: 10.1115/1.4039201]

43. Hall M, Frank E, Holmes G, Pfahringer B, Reutemann P, Witten IH. The WEKA data mining software: an update. SIGKDD Explor 2009;11(1):10-18. [doi: 10.1145/1656274.1656278] 
44. Sheng B, Deng C, Wang YH, Tang LH. System Analysis by Mapping a Fault-Tree Into a Bayesian-Network. In: Proceedings of the 3rd International Conference on Smart Engineering Materials. 2018 Presented at: IOP Conference Series; March 7-9, 2018; Bucharest, Romania.

45. Fenton NE, Neil M, Caballero JG. Using ranked nodes to model qualitative judgments in Bayesian networks. IEEE T Knowl Data En 2007 Oct;19(10):1420-1432. [doi: 10.1109/TKDE.2007.1073]

46. BayesFusion. 2019. GeNIe Modeler User Manual URL: https://support.bayesfusion.com/docs/GeNIe.pdf [accessed 2019-01-31] [WebCite Cache ID 75pHVUhR7]

47. Altman RD. Criteria for the classification of osteoarthritis of the knee and hip. Scand J Rheumatol Suppl 1987;65:31-39. [doi: 10.3109/03009748709102175] [Medline: 3317807]

48. Jin C, Yang Y, Xue ZJ, Liu KM, Liu J. Automated analysis method for screening knee osteoarthritis using medical infrared thermography. J Med Biol Eng 2013;33(5):471-477. [doi: 10.5405/jmbe.1054]

49. Binol H, Cukur H, Bal A. A supervised discriminant subspaces-based ensemble learning for binary classification. Int $\mathrm{J}$ Adv Comput Res 2016;6(27):209-214. [doi: 10.19101/IJACR.2016.627008]

50. Alentorn-Geli E, Samuelsson K, Musahl V, Green CL, Bhandari M, Karlsson J. The association of recreational and competitive running with hip and knee osteoarthritis: a systematic review and meta-analysis. J Orthop Sports Phys Ther 2017 Jun;47(6):373-390. [doi: 10.2519/jospt.2017.7137] [Medline: 28504066]

51. Seni G, Elder JF. Ensemble methods in data mining: improving accuracy through combining predictions. Synthesis Lect Data Mining Knowl Disc 2010;2(1):1-126. [doi: 10.2200/S00240ED1V01Y200912DMK002]

52. Hawkins DM. The problem of overfitting. J Chem Inf Comput Sci 2004;44(1):1-12. [doi: 10.1021/ci0342472] [Medline: 14741005]

53. Peat G, Thomas E, Duncan R, Wood L, Hay E, Croft P. Clinical classification criteria for knee osteoarthritis: performance in the general population and primary care. Ann Rheum Dis 2006 Oct;65(10):1363-1367 [FREE Full text] [doi: 10.1136/ard.2006.051482] [Medline: 16627539]

54. Lappenschaar M, Hommersom A, Lucas PJ, Lagro J, Visscher S. Multilevel Bayesian networks for the analysis of hierarchical health care data. Artif Intell Med 2013 Mar;57(3):171-183. [doi: 10.1016/j.artmed.2012.12.007] [Medline: 23419697]

55. Norsys Software Corp. 2019. Introduction to Bayes Nets URL: https://www.norsys.com/tutorials/netica/secA/tut A1.htm [accessed 2019-01-31] [WebCite Cache ID 75pGwqf1G]

\author{
Abbreviations \\ AUC: area under the curve \\ BMI: body mass index \\ BN: Bayesian network \\ BRT: body reaction time \\ BS: Bayesian Search \\ DT: decision tree \\ EM: expectation-maximization \\ HBN: handcrafted BN \\ KNN: k-nearest neighbor \\ LEP: leg extension power \\ LR: logistic regression \\ MRI: magnetic resonance imaging \\ MSR: modified sit and reach \\ NPV: negative predictive value \\ OA: osteoarthritis \\ PPV: positive predictive value \\ SEBT: Star Excursion Balance Test \\ SHBN: semihandcrafted BN \\ SLSB: single-leg stance balance \\ TUG: Timed Up and Go test \\ WHR: waist-to-hip ratio
}


Edited by C Lovis; submitted 30.01.19; peer-reviewed by M Bjelogrlic, T Jiang, X Montet; comments to author 08.04.19; revised version received 21.05.19; accepted 31.05.19; published 18.07.19

Please cite as:

Sheng B, Huang L, Wang X, Zhuang J, Tang L, Deng C, Zhang $Y$

Identification of Knee Osteoarthritis Based on Bayesian Network: Pilot Study

JMIR Med Inform 2019;7(3):e13562

URL: http://medinform.jmir.org/2019/3/e13562/

doi: $10.2196 / 13562$

PMID: $\underline{31322132}$

(CBo Sheng, Liang Huang, Xiangbin Wang, Jie Zhuang, Lihua Tang, Chao Deng, Yanxin Zhang. Originally published in JMIR Medical Informatics (http://medinform.jmir.org), 18.07.2019. This is an open-access article distributed under the terms of the Creative Commons Attribution License (https://creativecommons.org/licenses/by/4.0/), which permits unrestricted use, distribution, and reproduction in any medium, provided the original work, first published in JMIR Medical Informatics, is properly cited. The complete bibliographic information, a link to the original publication on http://medinform.jmir.org/, as well as this copyright and license information must be included. 